\title{
Effect of acupuncture on memory function in old rats
}

\author{
In-Ho Choi, Hyung-Ho Lim \\ College of Korean Medicine, Gachon University
}

\begin{abstract}
Objectives: We investigated the effect of acupuncture on memory function in relation with neurogenesis in old rats. Methods: In this study, a step-down avoidance task for short-term memory and Y-maze task for spatial memory capability were conducted. Western blot analysis for brain-derived neurotorphic factor (BDNF) and tyrosine kinase B (TrkB), and immunohistochemistry for 5-bromo-2'-deoxyuridine (BrdU) were performed.

Results: Short-term memory and spatial memories were decreased in the old-aged rats. Expressions of BDNF and TrkB in the hippocampus were significantly decreased in the old-aged rats. Neurogenesis in the hippocampal dentate gyrus was also decreased in the old-aged rats. However, acupuncture treatment alleviated impairment of short-term and spatial memories induced by ageing. Acupuncture also increased the expressions of BDNF and TrkB and enhanced neurogenesis in the hippocampus. The present study showed that acupuncture alleviated ageing-induced short-term and spatial memory loss by increasing of BDNF and neurogenesis. Acupuncture at ST41-acupoint showed most potent effect than at ST36-acupoint or non-acupoint.

Conclusions: Acupuncture might be used as the effective therapeutic modality to ameliorate the age-related decrease of brain functions.
\end{abstract}

Key Words : Aging, acupuncture, neurogenesis, brain-derived neurotorphic factor, short-term memory, spatial memory

\section{Introduction}

As increase of life span, age-related cognitive disorders become a major health problem in humans. Ageing induces cognitive impairments, mainly memory performance ${ }^{1,2)}$. Ageing also leads to functional changes of the hippocampus, the brain area that plays a pivotal role in learning and memory process $^{3)}$. The hippocampus is also one of the limited brain areas in which neurogenesis occurs ${ }^{4,5)}$.

Neurogenesis occurs within the adult dentate gyrus of the hippocampal formation, and newly born neurons recruit into the preexistent neuronal circuits.
Neurogenesis is involved in hippocampal-dependent learning processes. Brain-derived neurotrophic factor (BDNF) promotes survival and differentiation of selected neuronal population. In the central nerve system (CNS), high levels of BDNF mRNA and protein were found in the hippocampus and cerebral cortex, both are target areas for basal forebrain cholinergic neurons involved in learning and memory processes ${ }^{6,7}$. Tyrosine kinase B (TrkB) is the high affinity catalytic receptor for several neurotrophins that are small protein growth factors that induce the survival and differentiation of distinct cell populations ${ }^{8)}$. The neurotrophins that activate TrkB

\footnotetext{
- Received : 8 May 2017

- Revised : 8 June 2017

- Accepted : 8 June 2017

- Correspondence to : Hyung-Ho Lim

College of Korean Medicine, Gachon University

1342, Seongnam-daero, Sujeong-gu, Seongnam-si, Gyeonggi-do, Korea

Tel : +8231-750-8599, Faxl : +82-31-750-5416, E-mail : omdlimhh@gachon.ac.kr
} 
are BDNF, neurotrophin-3, and neurotrophin-4. Of these, TrkB mediates the multiple effects of these neurotrophic factors, which include neuronal differentiation and survival ${ }^{9}$.

Acupuncture, a core component of traditional oriental medicine, is becoming recognized as an effective way for the treatment of many chronic diseases including pain, musculoskeletal diseases, drug addiction, stroke rehabilitation, and asthma ${ }^{10-12)}$. Liu et al.'s study ${ }^{13)}$ reported that acupuncture might exert protective effects on cognitive deficits and oxidative stress in cerebral multi-infarction rats. Acupuncture improved cognitive deficits and regulated brain cell proliferation in Alzheimer's disease model of mice ${ }^{14)}$.

The neuroprotective effects of acupuncture on several neurodegenerative diseases are well documented, however the effects of acupuncture on age-related memory deficits are not established yet. Thus, the purpose of this study was to ascertain the effects of acupuncture on short-term and spatial memories of old-aged rats in relation with neurogenesis in the hippocampal dentate gyrus. For this study, a step-down inhibitory avoidance task and Y-maze task for memory functions, BDNF and TrkB western blot for neurotrophins expressions, 5-bromo-2'-deoxyuridine (BrdU) immunohistochemistry for neurogenesis were conducted.

\section{Materials and methods}

\section{Animals}

Sprague-Dawley adult male rats were obtained from a commercial breeder (Orient Co., Seoul, Korea) for the experiment. The experimental procedures were performed in accordance with the animal care guidelines of the National Institute of Health (NIH) and the Korean Academy of Medical Sciences. Each animal was housed under controlled temperature $\left(23 \pm 2^{\circ} \mathrm{C}\right)$ and lighting $(08: 00 \mathrm{~h}-20: 00 \mathrm{~h})$ conditions with food and water made available ad libitum. Two age groups of rats were used: 7 weeks old rats $(210 \pm 5 \mathrm{~g})$ for young-aged group and 18 months old rats $(480 \pm 15 \mathrm{~g})$ for old-aged group. The animals were randomly divided into five groups $(\mathrm{n}=10$ in each group): the young-aged group, the old-aged control group, the old-aged and ST36-acupoint-acupunctured group, the old-aged and ST41-acupoint-acupunctured group, and the old aged and non-acupoint-acupunctured group.

All rats received $50 \mathrm{mg} / \mathrm{kg} \quad \mathrm{BrdU}$ (Sigma Chemical Co., St. Louis, MO, USA) intraperitoneally once a day for 7 consecutive days, starting one day after acupuncture treatment.

\section{Acupunctural treatment}

The animals in the acupunctured groups received acupuncture bilaterally at respective site, once a day for 5 weeks. ST36-acupoint (Zusanli) is located at the proximal one fifth points on the line from the patella ligament to the anterior side of ankle crease. ST41-acupoint (Jiexi) is located at the depression between tibialis anterior tendon and extensor digitorum longus tendon, on the anterior side of ankle crease. Non-acupoint is located at the side of the hip. For acupunctural stimulation, after insertion of stainless acupuncture needles $(0.3 \mathrm{~mm})$, the needles were manually rotated clockwise and counter-clockwise 30 times in each leg to increase the efficiency of acupuncture and kept in place for $10 \mathrm{~min}$ (Fig. 1).

\section{Step-down avoidance task}

The latency of the step-down avoidance task was determined to evaluate short-term memory capability. The rats were trained in a step-down avoidance task at 34 days after starting of acupuncture treatment. One hour after training, the latency (sec) of the animals in each group was determined. The rats were placed on a $7 \times 25 \mathrm{~cm}$ platform $2.5 \mathrm{~cm}$ high. The platform faced a $42 \times 25 \mathrm{~cm}$ grid of parallel 0.1 

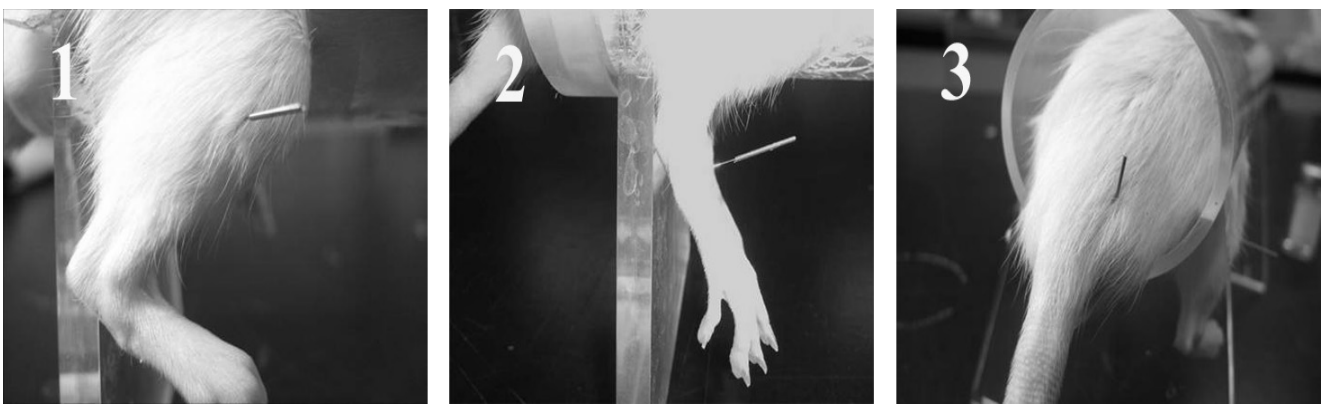

Fig. 1. The locations of the acupoints in rats. (1) ST36-acupoint, (2) ST41-acupoint, (3) non-acupoint.

cm-caliber stainless steel bars spaced $1 \mathrm{~cm}$ apart. In training sessions, the animals received $0.5 \mathrm{~mA}$, scramble foot shock for $2 \mathrm{sec}$ immediately upon stepping down. The interval of rats stepping down and placing all four paws on the grid was defined as the latency time. The latency over $300 \mathrm{sec}$ was counted as $300 \mathrm{sec}$.

\section{Y-maze task}

The percentage of alternation in the Y-maze task was determined to evaluate spatial memory capability. The rats were tested one day after determining the step-down avoidance task. The arm-recognition component of the Y-maze had three identical arms, $50 \mathrm{~cm}$ long, $12 \mathrm{~cm}$ wide, and $35 \mathrm{~cm}$ high. The floor of the maze was covered with soiled animal bedding. During Y-maze test, the rats were placed in the center of a symmetrical Y-maze and were allowed to explore freely in the maze for $8 \mathrm{~min}$. The total number and sequence of arms entered were recorded. An arm was entered if the hind paws of the rat were completely in the arm. The percentage alternation that was the percentage of the number of entry into all three arms in the maximum possible alternations (the total number of arms entered divided by 3 ) was calculated for each rat.

\section{Tissue preparation}

The rats were sacrificed immediately after determining the behavioral test. The animals were anesthetized using Zoletil $50^{\circledR}(10 \mathrm{mg} / \mathrm{kg}$, i.p.; Vibac Laboratories, Carros, France), transcardially perfused with $50 \mathrm{mM}$ phosphate-buffered saline (PBS), and fixed with a freshly prepared solution consisting of $4 \%$ paraformaldehyde in $100 \mathrm{mM}$ phosphate buffer ( $\mathrm{PB}, \mathrm{pH}$ 7.4). Brains were dissected, post-fixed in the same fixative overnight, and transferred to $30 \%$ sucrose for cryoprotection. Forty $\mu \mathrm{m}$ thick coronal sections were made using a freezing microtome (Leica, Nussloch, Germany). Ten slice sections on average in the dentate gyrus region were collected from each rat. The sections from $2.5 \mathrm{~mm}$ to $2.7 \mathrm{~mm}$ posterior of the bregma were used for immunohistochemistry.

\section{BDNF and TrkB Western blot analysis}

The hippocampal tissues were collected, and then were immediately frozen at $-80^{\circ} \mathrm{C}$. The hippocampal tissues were homogenized on ice, and lysed in a lysis buffer containing $50 \mathrm{mM}$ Tris- $\mathrm{HCl}$ (pH 7.5), $150 \mathrm{mM} \mathrm{NaCl}, 0.5 \%$ deoxycholic acid, $1 \%$ Nonidet P40, $0.1 \%$ SDS, $1 \mathrm{mM}$ PMSF, and $100 \mathrm{mg} / \mathrm{ml}$ leupeptin. Protein content was measured using a Bio-Rad colorimetric protein assay kit (Bio-Rad, Hercules, CA, USA). Protein of $30 \mu \mathrm{g}$ was separated on SDS-polyacrylamide gels and transferred onto a nitrocellulose membrane. Mouse actin antibody (1:500; Santa Cruz Biotechnology, Santa Cruz, CA, 
USA), rabbit BDNF antibody, and rabbit TrkB antibody (1:1000; Santa Cruz Biotechnology) were used as the primary antibodies. Horseradish peroxidase -conjugated anti-rabbit antibody for BDNF and TrkB (1:2000; Vector Laboratories, Burlingame, CA. USA) was used as the secondary antibodies. Experiment was performed in normal lab conditions and at room temperature except membrane transfer. Membrane transfer was performed at $4^{\circ} \mathrm{C}$ with the cold pack and prechilled buffer. Band detection was performed using the enhanced chemiluminescence (ECL) detection kit (Santa Cruz Biotechnology).

\section{BrdU immunohistochemistry}

Slice sections were first permeabilized by incubating in $0.5 \%$ Triton X-100 in PBS for $20 \mathrm{~min}$, then pretreated in $50 \%$ formamide- $2 \mathrm{x}$ standard saline citrate (SSC) at $65^{\circ} \mathrm{C}$ for $2 \mathrm{~h}$, denatured in 2 $\mathrm{N} \mathrm{HCl}$ at $37^{\circ} \mathrm{C}$ for $30 \mathrm{~min}$, and rinsed twice in 100 $\mathrm{mM}$ sodium borate ( $\mathrm{pH} 8.5$ ). Afterwards, the sections were incubated overnight at $4{ }^{\circ} \mathrm{C}$ with $\mathrm{BrdU}$-specific mouse monoclonal antibody (1:600; Roche, Mannheim, Germany). The sections were then washed three times with PBS and incubated with biotinylated mouse secondary antibody (1:200; Vector Laboratories) for $1 \mathrm{~h}$. The sections were then incubated for another $1 \mathrm{~h}$ with an avidin-peroxidase complex (1:100; Vector Laboratories). For visualization, sections were incubated in $50 \mathrm{mM}$ Tris- $\mathrm{HCl}(\mathrm{pH}$ 7.6) containing $0.03 \% \mathrm{DAB}, 40 \mathrm{mg} / \mathrm{ml}$ nickel chloride, and $0.03 \%$ hydrogen peroxide for $5 \mathrm{~min}$.

After BrdU staining, differentiation of $\mathrm{BrdU}$ -positive cells was determined on the same section using a mouse anti-neuronal nucleic (NeuN) antibody (1:1000; Chemicon International, Temecula, CA, USA). The sections were washed three times with PBS, incubated for $1 \mathrm{~h}$ with a biotinylated anti-mouse secondary antibody. For staining, sections were incubated in a reaction mixture consisting of $0.03 \%$ $\mathrm{DAB}$ and $0.03 \%$ hydrogen peroxide for $5 \mathrm{~min}$. The sections were finally mounted onto gelatin-coated slides. The slides were air-dried overnight at room temperature, and coverslips were mounted using Permount $^{\circledR}$ (Fisher Scientific Inc., Waltham, MA, USA).

\section{Data analysis}

The number of BrdU-positive cells in the dentate gyrus was counted. The area of the dentate gyrus was measured by Image-Pro ${ }^{\circledR}$ Plus image analysis system (Media Cyberbetics Inc., Silver Spring, MD, USA) attached to a light microscope (Olympus, Tokyo, Japan). The number of BrdU-positive cells in the dentate gyrus was counted hemilaterally through a light microscope (Olympus). The number of BrdU-positive cells was expressed as the number of cells per square millimeter in the dentate gyrus. To compare relative expression of BDNF and TrkB proteins, detected bands were calculated densitometrically using Molecular Analyst ${ }^{\mathrm{TM}}$ version 1.4.1 (Bio-Rad).

Statistical analysis was performed using one-way ANOVA followed by Duncan's post-hoc test, and the results are expressed as the mean \pm standard error of the mean (S.E.M.). Significance was set as $p<0.05$.

\section{Results}

\section{Effect of acupuncture on short-term memory in step-down avoidance task}

The latencies of the step-down avoidance task are presented in Fig. 2. The latency time was 267.00 $\pm 26.90 \mathrm{sec}$ in the young-aged group, $13.90 \pm 2.29 \mathrm{sec}$ in the old-aged and control group, $50.50 \pm 11.58 \mathrm{sec}$ in the old-aged and ST36-acupoint-acupunctured group, $81.60 \pm 17.74 \mathrm{sec}$ in the old-aged and ST4 1-acupoint-acupunctured group, and 20.00 $\pm 7.74 \mathrm{sec}$ in the old-aged and non-acupoint-acupunctured group.

These results showed that latency time of the old-aged groups was conspicuously shorter than that of the young-aged group. In contrast, acupuncture 


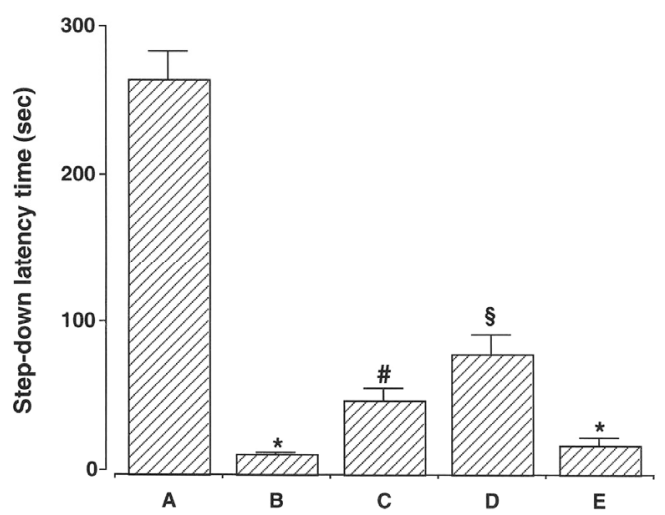

Fig. 2. Effect of acupuncture on latency of a step-down avoidance task. (A) Young-aged group, (B) old-aged control group, (C) old-aged and ST36-acupoint -acupunctrued group, (D) old-aged and ST41 -acupoint-acupunctured group, (E) old-aged and non-acupoint-acupunctured group. *represents $p<$ 0.05 compared to the young-aged group. \# represents $p<0.05$ compared to the old-aged control group. $\S$ represents $p<0.05$ compared to the old-aged and ST36-acupoint-acupunctrued group.

treatment alleviated ageing-induced decrease of short-term memory. Especially, acupuncture at ST41 acupoint showed most potent alleviating effect on short-term memory loss induced by ageing.

\section{Effect of acupuncture on spatial memory in Y-maze task}

The percentages of alternation in the Y-maze task are presented in Fig. 3. The percentage of alternation was $41.23 \pm 0.65 \%$ in the young-aged group, 23.60 $\pm 2.68 \%$ in the old-aged and control group, 28.49 $\pm 2.74 \%$ in the old aged-and ST36-acupoint-acupunctured group, $30.56 \pm 1.96 \%$ in the old-aged and ST41-acupoint -acupunctured group, and $22.46 \pm 3.40 \%$ in the old-aged and non-acupoint-acupunctured group.

These results showed that percentage of alternation in the old-aged groups was conspicuously decreased than that of the young-aged group. In contrast, acupuncture treatment alleviated ageing-induced decrease of spatial memory. Especially, acupuncture

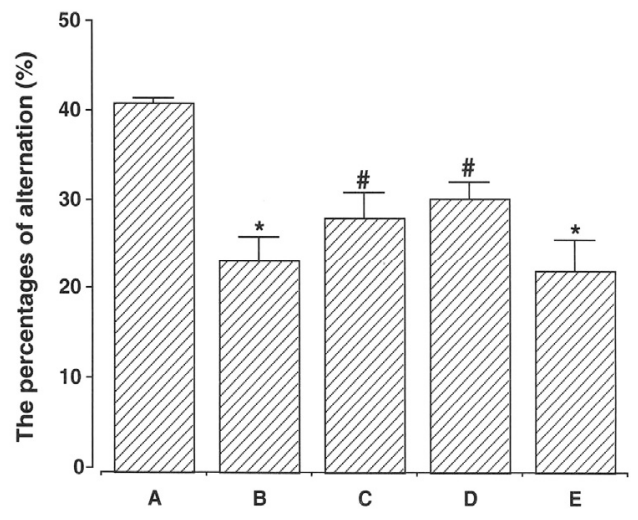

Fig. 3. Effect of acupuncture on percentages of alternation in the Y-maze task. (A) Young-aged group, (B) old-aged control group, (C) old-aged and ST36 -acupoint-acupunctrued group, (D) old-aged and ST41-acupoint-acupunctured group, (E) old-aged and non-acupoint-acupunctured group. * represents $p<0.05$ compared to the young-aged group. \# represents $p<0.05$ compared to the old-aged control group.

at ST35- acupoint and ST41-acupoint showed more potent alleviating effect on spatial memory loss induced by ageing.

\section{Effect of acupuncture on BDNF and TrkB expressions}

We ascertained the expression of BDNF (14 kDa), a member of the nerve growth factor family of neurotrophins. The expression of the BDNF protein was $3.26 \pm 0.18$ in the young- aged group, $2.00 \pm 0.29$ in the old-aged and control group, $2.34 \pm 0.23$ in the old-aged and ST36-acupoint-acupunctured group, $2.81 \pm 0.31$ in the old-aged and ST41-acupoint -acupunctured group, and $1.44 \pm 0.07$ in the old-aged and non-acupoint-acupunctured group.

We also ascertained the expression of TrkB (95-145 kDa), neurotrophic receptor for BDNF. The expression of the $\mathrm{TrkB}$ protein was $2.61 \pm 0.04$ in the young-aged group, $1.43 \pm 0.03$ in the old-aged and control group, $1.80 \pm 0.06$ in the old-aged and ST36 

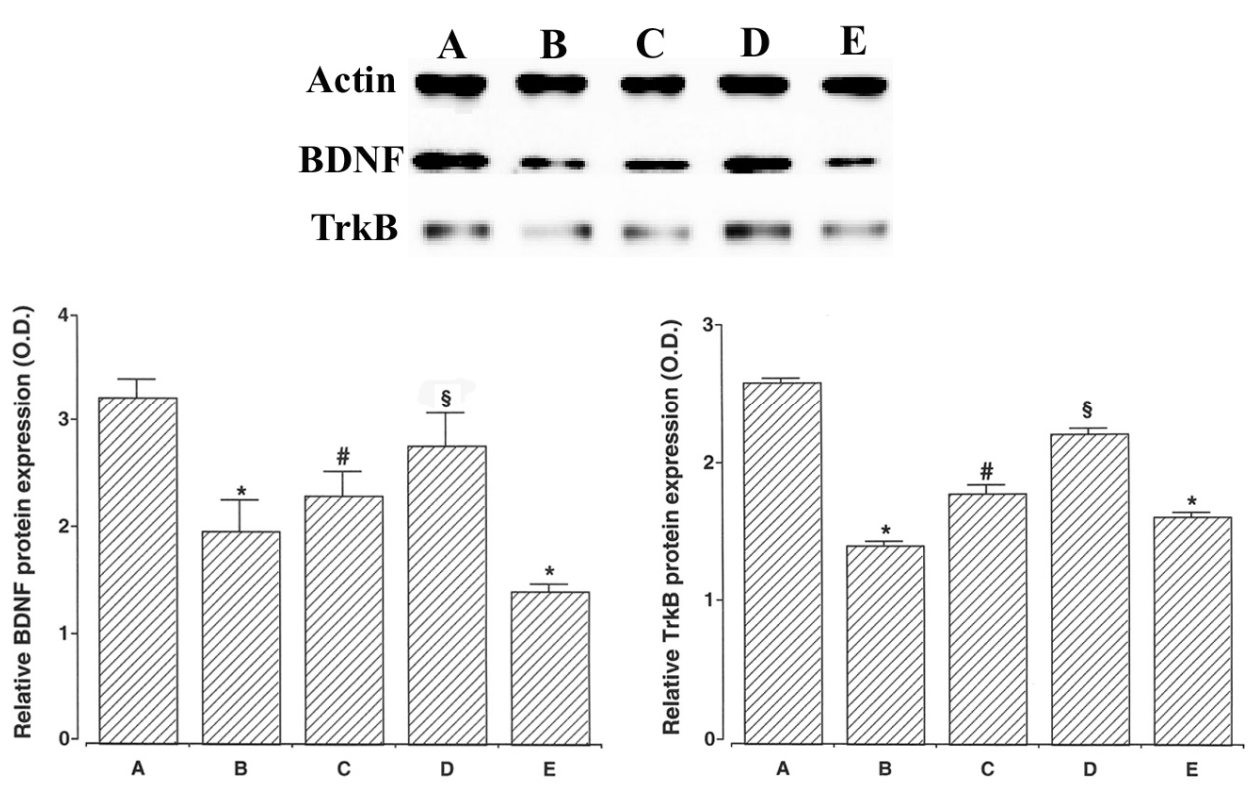

Fig. 4. Effect of acupuncture on the expressions of BDNF and TrkB. Actin was used as an internal control (46 kDa). (A) Young-aged group, (B) old-aged and control group, (C) old-aged and ST36-acupoint-acupunctrued group, (D) old-aged and ST41-acupoint-acupunctured group, (E) old-aged and non-acupoint-acupunctured group. * represents $p<0.05$ compared to the young-aged group. \# represents $p<0.05$ compared to the old-aged control group. $\S$ represents $p<0.05$ compared to the old-aged and ST36-acupoint-acupunctrued group.

-acupoint-acupunctured group, $2.23 \pm 0.04$ in the old-aged and ST41-acupoint-acupunctured group, $1.63 \pm 0.03$ in the old-aged and non-acupoint -acupunctured group.

The expressions of BDNF and TrkB in the old-aged group were significantly lower than in the young-aged group. In contrast, acupuncture treatment remarkably enhanced the BDNF and TrkB expressions in the old-aged rats. Especially, acupuncture at ST41-acupoint showed most potent enhancing effect on BDNF and TrkB expressions.

\section{Effect of acupuncture on neurogenesis in the hippocampal dentate gyrus}

Photomicrographs of BrdU-positive cells in the hippocampal dentate gyrus are presented in Fig. 4. The number of BrdU-positive cells was $245.92 \pm$ $32.75 / \mathrm{mm}^{2}$ in the young-aged group, $48.29 \pm 5.39 / \mathrm{mm}^{2}$ in the old-aged and control group, $93.41 \pm 12.06 / \mathrm{mm}^{2}$ in the old-aged and ST36-acupoint-acupunctured group, $130.07 \pm 9.66 / \mathrm{mm}^{2}$ in the old-aged and ST 41 -acupoint-acupunctured group, $48.29 \pm 6.04 / \mathrm{mm}^{2}$ in the old-aged and non-acupoint-acupunctured group.

These results showed that neurogenesis in the dentate gyrus of old-aged group was significantly decreased than that of the young-aged group. In contrast, acupuncture treatment exerted enhancing effect of neurogenesis in the hippocampal dentate gyrus in the old-aged rats. Especially, acupuncture at ST41-acupoint showed most potent enhancing effect on neurogenesis.

\section{Discussion}

Ageing process accompanies with cognitive impairments, particularly memory $\operatorname{loss}^{1,2,15}$. In previous study, episodic memory, short-term memory, 


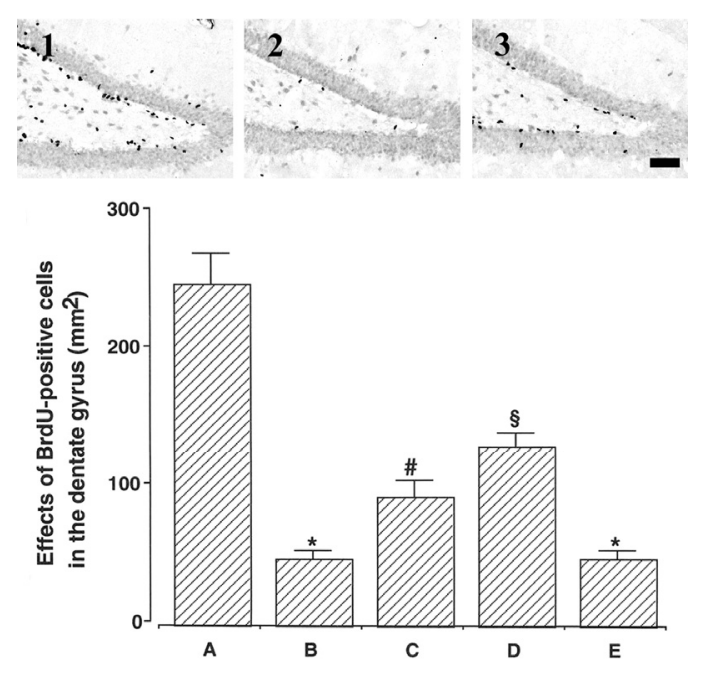

Fig. 5. Effect of treadmill exercise on neurogenesis in the dentate gyrus. Upper: Photomicrographs of 5-bromo -2 -deoxyuridine (BrdU) positive cells in the dentate gyrus. (1) Young-aged group, (2) old-aged control group, (3) old-aged and ST41-acupoint-acupunctured group. The sections were stained for BrdU (black) and neuronal nuclei (NeuN; brown). The scale bar represents $400 \mu \mathrm{m}$. Lower: The number of BrdU -positive cells in each group. (A) Young-aged group, (B) old-aged and control group, (C) old-aged and ST36-acupoint-acupunctrued group, (D) old-aged and ST41-acupoint-acupunctured group, (E) old-aged and non-acupoint-acupunctured group. *represents $p<0.05$ compared to the young -aged group. \# represents $p<0.05$ compared to the old-aged control group. $\S$ represents $p<0.05$ compared to the old-aged and ST36-acupoint -acupunctrued group.

and spatial memory were decreased during normal ageing process ${ }^{16)}$.

In the present result, the behavioral tests revealed that ageing incapacitated short-term memory and spatial memory abilities. Ageing-induced memory loss may be related to impairment of the ability to refresh recently processed information. Age-dependent spatial memory impairment is known to be related with the alteration of hippocampal plasticity and neurogenesis ${ }^{17,18)}$.

BDNF expression in the hippocampus is closely associated with memory consolidation and synaptic plasticity. Hippocampus is the brain region that has the highest expression of BDNF and its TrkB receptor ${ }^{19)}$. In contrast, a decrease of BDNF expression is associated with neuronal atrophy or death, for instance, with ageing or in some neurological disorders $^{19)}$. Furthermore, the deficit of BDNF induced impairment of hippocampal long-term potentiation, and this deficit in synaptic function amended by the exogenous application or over-expression of $\mathrm{BDNF}^{20,21)}$.

Our present study showed that the abundant expressions of BDNF and TrkB in the hippocampus were observed in the young-aged rats, whereas the old-aged rats showed significantly low expressions of BDNF and TrkB in the hippocampus. Decreased expressions of BDNF and TrkB by ageing are also associated with the loss of short-term and spatial memories in the old-aged rats.

The newly born cells are preferentially recruited into circuits supporting spatial memory formation, and adult neurogenesis in the hippocampus plays a critical role in the hippocampus-dependent learning process $^{22)}$. Ageing causes a dramatic decrease in neurogenesis, and this change might induce age-dependent cognitive dysfunction ${ }^{18,23,24)}$.

In present study, the number of BrdU-positive cells in the hippocampal dentate gyrus was significantly decreased in the old-aged rats, indicating that ageing decreased neurogenesis in the hippocampal dentate gyrus. In the old-aged rats, short-term and spatial memories were also decreased, in this study.

Stimulation of acupoint located in meridians has been proven to activate specific areas of the human brains ${ }^{25}$. Especially, acupuncture produced extensive deactivation of hippocampus in the limbic system ${ }^{25,26)}$. Thus, acupuncture may mediate its anti-pain, anti-anxiety, and other therapeutic effects via this intrinsic neural circuit. Activation of intrinsic neural circuit by acupuncture plays a central role in the affective and cognitive dimensions of pain as well as in the regulation and integration of emotion, memory 
processing, and immunological function ${ }^{26)}$. We used acupoint, named Zusanli (ST36), Jiexi (ST41). In fMRI experiment, acupuncture stimulation on both acupoints ST36 and ST41 evoked activation at the ipsilateral middle frontal gyrus and hippocampus ${ }^{27,28)}$. In addition, Yun et al.'s study ${ }^{29)}$ reported that acupuncture may relieve stress by modulating neurotrophic factor expression. In neuropathological model research, acupuncture therapy may enhance expressions of BDNF protein and BDNF mRNA in the Parkinson's disease and stroke model ${ }^{30,31)}$.

Our present study showed that acupuncture significantly enhanced BDNF and TrkB protein expressions in the hippocampus the old-aged rats. Acupuncture at ST41-acupoint appeared more potent enhancing effect compared to the ST36-acupoint or non-acupoint.

Improved spatial memory was correlated with the increased BDNF mRNA expression in the hippocampus of the rats ${ }^{32}$. In addition, adult hippocampal neurogenesis is not only affected by the external stimuli but also regulated by the internal growth factors, including BDNF. The neurogenesis has been suggested as the novel mechanism that enhance memory function.

In present study, neurogenesis in the hippocampal dentate gyrus was significantly increased by acupuncture in old-aged rats. Acupuncture at ST41-acupoint appeared more potent increasing effect compared to the ST36-acupoint or non-acupoint.

In the behavioral tests, acupuncture improved short-term and spatial memories in old-aged rats. Acupuncture at ST41-acupoint appeared more potent improving effect compared to the ST36-acupoint or non-acupoint.

This study showed that acupuncture alleviated ageing-induced deterioration of short-term and spatial memories by increasing of BDNF expression and neurogenesis. In this study, acupuncture at ST41 -acupoint showed most potent effect than at ST36-acupoint or non-acupoint. Based on the present results, acupuncture might be used as the effective therapeutic modality to ameliorate the age-related decrease of brain functions.

\section{References}

1. Colombo PJ, Gallagher M. Individual differences in spatial memory and striatal ChAT activity among young and aged rats. Neurobiol Learn Mem. 1998;70:314-327.

2. Nyffeler M, Yee BK, Feldon J, Knuesel I. Abnormal differentiation of newborn granule cells in age-related working memory impairments. Neurobiol Aging. 2010;31:1956-1974.

3. Mattson MP, Magnus T. Ageing and neuronal vulnerability. Nat Rev Neurosci. 2006;7:278-294.

4. Gould E, Tanapat P, Hastings NB, Shors TJ. Neurogenesis in adulthood: a possible role in learning. Trends Cogn Sci. 1999;3:186-192.

5. Leuner B, Kozorovitskiy Y, Gross CG, Gould E. Diminished adult neurogenesis in the marmoset brain precedes old age. Proc Natl Acad Sci USA. 2007;23:17169-17173.

6. Yan Q, Rosenfeld RD, Matheson CR, Hawkins $\mathrm{N}$, Lopez OT, Bennett L, et al. Expression of brain-derived neurotrophic factor protein in the adult rat central nervous system. Neuroscience. 1997;78:431-448.

7. Cirulli F, Berry A, Chiarotti F, Alleva E. Intrahippocampal administration of BDNF in adult rats affects short-term behavioral plasticity in the Morris water maze and performance in the elevated plus-maze. Hippocampus. 2004;14: 802-807.

8. Naylor RL, Robertson AG, Allen SJ, Sessions RB, Clarke AR, Mason GG, et al. A discrete domain of the human TrkB receptor defines the binding sites for BDNF and NT-4. Biochem Biophys Res Commun. 2002;291:501-507.

9. Tyler WJ, Alonso M, Bramham CR, Pozzo-Miller LD. From acquisition to consolidation: on the role of brain-derived neurotrophic factor signaling 
in hippocampal-dependent learning. Learn Mem. 2002;9:224-237.

10. Kim EH, Kim YJ, Lee HJ, Huh Y, Chung JH, Seo JC, et al. Acupuncture increases cell proliferation in dentate gyrus after transient global ischemia in gerbils. Neurosci Lett. 2001; 30:21-24.

11. Vickers A, Wilson P, Kleijnen J. Acupuncture. Qual Saf Health Care. 2002;11:92-97.

12. Madsen MV, Gøtzsche PC, Hróbjartsson A. Acupuncture treatment for pain: systematic review of randomised clinical trials with acupuncture, placebo acupuncture, and no acupuncture groups. BMJ. 2009;27:338-349.

13. Liu CZ, Yu JC, Zhang XZ, Fu WW, Wang T, Han JX. Acupuncture prevents cognitive deficits and oxidative stress in cerebral multi-infarction rats. Neurosci Lett. 2006;23:45-50.

14. Cheng H, Yu J, Jiang Z, Zhang X, Liu C, Peng $\mathrm{Y}$, et al. Acupuncture improves cognitive deficits and regulates the brain cell proliferation of SAMP8 mice. Neurosci Lett. 2008;20:111 -116 .

15. Ingram DK, Spangler EL, Iijima $\mathrm{S}$, Kuo $\mathrm{H}$, Bresnahan EL, Greig NH, et al. New pharmacological strategies for cognitive enhancement using a rat model of age-related memory impairment. Ann N Y Acad Sci. 1994;30:16-32.

16. Nilsson OG, Gage FH. Anticholinergic sensitivity in the aging rat septohippocampal system as assessed in a spatial memory task. Neurobiol Aging. 1993;14:487-497.

17. Drapeau E, Mayo W, Aurousseau C, Le Moal M, Piazza PV, Abrous DN. Spatial memory performances of aged rats in the water maze predict levels of hippocampal neurogenesis. Proc Natl Acad Sci USA. 2003;100:14385-14390.

18. Drapeau E, Montaron MF, Aguerre S, Abrous DN. Learning-induced survival of new neurons depends on the cognitive status of aged rats. J
Neurosci. 2007;30:6037-6044.

19. Murer MG, Yan Q, Raisman-Vozari R. Brain -derived neurotrophic factor in the control human brain, and in Alzheimer's disease and Parkinson's disease. Progr Neurobiol. 2001;63: 71-124.

20. Korte M, Carroll P, Wolf E, Brem G, Thoenen $\mathrm{H}$, Bonhoeffer T. Hippocampal long-term potentiation is impaired in mice lacking brain-derived neurotrophic factor. Proc Natl Acad Sci USA. 1995;12:8856-8860.

21. Patterson SL, Abel T, Deuel TA, Martin KC, Rose JC, Kandel ER. Recombinant BDNF rescues deficits in basal synaptic transmission and hippocampal LTP in BDNF knockout mice. Neuron. 1996;16:1137-1145.

22. Kee N, Teixeira CM, Wang AH, Frankland PW. Preferential incorporation of adult-generated granule cells into spatial memory networks in the dentate gyrus. Nat Neurosci. 2007;10: 355-362.

23. Kuhn HG, Dickinson-Anson $\mathrm{H}$, Gage FH. Neurogenesis in the dentate gyrus of the adult rat: age-related decrease of neuronal progenitor proliferation. J Neurosci. 1996;15:2027-2033.

24. Rao MS, Hattiangady B, Shetty AK. The window and mechanisms of major age-related decline in the production of new neurons within the dentate gyrus of the hippocampus. Aging Cell. 2006;5:545-558.

25. Li L, Liu H, Li YZ, Xu JY, Shan BC, Gong D, et al. The human brain response to acupuncture on same-meridian acupoints: evidence from an fMRI study. J Altern Complement Med. 2008; 14:673-678.

26. Fang J, Jin Z, Wang Y, Li K, Kong J, Nixon $\mathrm{EE}$, et al. The salient characteristics of the central effects of acupuncture needling: limbic -paralimbic-neocortical network modulation. Hum Brain Mapp. 2009;30:1196-1206.

27. Wu MT, Hsieh JC, Xiong J, Yang CF, Pan HB, 
Chen YC, et al. Central nervous pathway for acupuncture stimulation: localization of processing with functional MR imaging of the brain --preliminary experience. Radiology. 1999;212: 133-141.

28. Hui KK, Liu J, Makris N, Gollub RL, Chen AJ, Moore CI, et al. Acupuncture modulates the limbic system and subcortical gray structures of the human brain: evidence from fMRI studies in normal subjects. Hum Brain Mapp. 2000;9: 13-25.

29. Yun SJ, Park HJ, Yeom MJ, Hahm DH, Lee HJ, Lee EH. Effect of electroacupuncture on the stress-induced changes in brain-derived neurotrophic factor expression in rat hippocampus. Neurosci Lett. 2002;25:85-88.
30. Jeon S, Kim YJ, Kim ST, Moon W, Chae Y, Kang M, et al. J. Proteomic analysis of the neuroprotective mechanisms of acupuncture treatment in a Parkinson's disease mouse model. Proteomics. 2008;8:4822-4832.

31. Kim WS, Kim IS, Kim SJ, Wei P, Choi DH, Han TR. Effect of electroacupuncture on motor recovery in a rat stroke model during the early recovery stage. Brain Res. 2009;12:176-183.

32. Falkenberg T, Mohammed AK, Henriksson B, Persson H, Winblad B, Lindefors N. Increased expression of brain-derived neurotrophic factor mRNA in rat hippocampus is associated with improved spatial memory and enriched environment. Neurosci Lett. 1992;138:153-156. 